\title{
The molecular underpinnings of fertility: genetic approaches in Caenorhabdities elegans.
}

\author{
Xue $\mathrm{Mei}^{1}$ and Andrew Singson ${ }^{1}$ \\ ${ }^{1}$ Rutgers The State University of New Jersey
}

July 9, 2020

\begin{abstract}
The study of mutations that impact fertility has a catch-22. Fertility mutants are often lost since they cannot simply be propagated and maintained. This has hindered progress in understanding the genetics of fertility. In mice, several molecules are found to be required for the interactions between the sperm and egg, with JUNO and IZUMO1 being the only known receptor pair on the egg and sperm surface, respectively. In C. elegans, a total of 12 proteins on the sperm or oocyte have been identified to mediate their interactions. Majority of these genes were identified through mutants isolated from genetic screens. In this review, we summarize the several key screening strategies that led to the identification of fertility mutants in C. elegans and provide a perspective about future research using genetic approaches. Recently, advancements in new technologies such as highthroughput sequencing and Crispr-based genome editing tools have accelerated the molecular, cell biological, and mechanistic analysis of fertility genes. We review how these valuable tools advance our understanding of the molecular underpinnings of C. elegans fertilization and complement fertility research in humans and other species.
\end{abstract}

\section{Hosted file}

XM Perspective genetic approach to understand fertility.pdf available at https://authorea. com/users/341344/articles/468279-the-molecular-underpinnings-of-fertility-geneticapproaches-in-caenorhabdities-elegans 